\title{
Three-dimensional Compressible Flow Simulation of Top-blown Multiple Jets in Converter
}

\author{
Wenjing WANG, ${ }^{1,2)}$ Zhangfu YUAN, ${ }^{1)}$ Hiroyuki MATSUURA, ${ }^{3)}$ Hongxin ZHAO, ${ }^{2)}$ Cheng DAl' and \\ Fumitaka TSUKIHASHI ${ }^{3)}$
}

1) Department of Energy and Resources Engineering, College of Engineering, Peking University, Haidian District, Beijing 100871 China. 2) State Key Laboratory of Multiphase and Complex Systems, Institute of Process Engineering, Chinese Academy of Sciences, Haidian District, Beijing 100190 China. 3) Department of Advanced Materials Science, Graduate School of Frontier Sciences, The University of Tokyo, 5-1-5 Kashiwanoha, Kashiwa, Chiba 277-8561 Japan.

E-mail: matsuura@k.u-tokyo.ac.jp

(Received on October 8, 2009; accepted on January 14, 2010)

\begin{abstract}
A three-dimensional mathematical model has been developed to simulate the compressible jets flow from the top-blown lance with multi-nozzles in converter to a free surrounding domain. The variations of fluid density and viscosity, high temperature, and Mach number were taken into consideration in this model which was validated against the physical modeling results. More specifically, computations were obtained to compare the widely used realizable $k-\varepsilon$ turbulence model against the standard $k-\omega$ turbulence model, which shows that the latter one is superior to calculate diverse turbulent conditions within the multiple jets. Moreover, the coalescence pattern of the multiple jets has been illustrated by their Mach number distribution, and each individual jet proceeds in a curve course, bending to the lance center and tending to unite. The effects of the inclination angles on the jets coalescence were also investigated, which indicates that the lower the inclination angle is, the stronger the interfering extent between the multiple jets is. With the help of this model, the dynamic power of the multiple jets to support the cavity formation was demonstrated, and additionally, a mathematical model concerning the effective penetration radius and digressing path of the multiple jets was proposed by taking the inclination angle and the axial distance from the nozzle tips as arguments.
\end{abstract}

KEY WORDS: BOF; top blowing; multiple jets; coalescence; inclination angle; CFD; turbulence model; three dimensional simulation.

\section{Introduction}

In the metallurgical process of steelmaking, the topblown jets through multi-nozzles, efficiently transforming the pressure energy at the nozzles-inlet into the kinetic energy, play an important role in stirring the metal bath, consequentially following surface deformation modes such as dimpling, splashing and penetrating. Therefore, jets behavior under their designed conditions, such as correct pressure, flow rate, and Mach number, is a crucial factor to obtain a good understanding of the underlying fluid dynamics in the BOF in order to optimize the involved kinetics. In many researches, ${ }^{1-5)}$ behaviors of the single jet through one nozzle or the multiple jets through the multi-nozzles have been investigated from an experimental point of view. With the help of Pitot tube, these experimental measurements are effective to directly know the jet behavior, for example the velocity field distribution, but under the condition of a small laboratory model it is very difficult to predict it on a commercial basis which is characteristic of more complex environment, such as high Mach number and high temperature.

Because the jet from the oxygen lance is one of the key process control tools available to engineer the steelmaking reactions within the BOF vessel, a more in-depth understanding into the characteristics of the supersonic jets under commercial conditions is warranted. As a result, there have been some efforts ${ }^{6-11)}$ in the field of computational fluid dynamics with the purpose of developing accurate and efficient numerical models to solve the jets flow in the commercial converter. Hatta et al. ${ }^{6}$ and Peng et $a l^{7)}$ both derived the system of mathematical equations to simulate the steady, quasi-one-dimensional supersonic flow through a single De Laval nozzle, which can be applied to the establishment of the conditions of optimum nozzles of performance. Tago et al. ${ }^{1)}$ investigated the characteristics of jets from single-nozzle and multi-nozzle lance under the condition that the ambient temperature was equal to the room temperature by a two-dimensional simulation based on fluid dynamics. Ersson et al. ${ }^{8,9)}$ fundamentally modeled the flow field and surface deformation caused by an impinging jet in a top blown reactor, but their general models get a relatively rough estimate because of its rather inhibited assumptions. Odenthal et al. ${ }^{10)}$ showed a compressible and steady simulation of the single jet through the Laval nozzle to illustrate the flow regimes in a supersonic jet. Sambasivam et al. ${ }^{11}$ ) put forward the numerical studies of the jet behaviors and their coalescence pattern in a seven-hole novel lance design.

Although the aforementioned CFD studies were useful to demonstrate the hidden and significant properties of the top-blowing jets to an extent, yet these researches mainly focused on the single jet behavior. There is still lack of a robust three-dimensional simulation model of multiple jets 
under the commercial conditions to analyses their essentials in detail, for example the jet coalescence pattern. As imagined, the jet coalescence, if it occurs, reduces the impact of the individual jet as the jets engulf a huge mass of ambient medium. Jet coalescence is thus considered detrimental. Furthermore, wide ranges of Reynolds and Mach numbers in the converter strengthen the complex characteristics of the jets. In order to study the pattern of the jets interference extent and their influence on the metal bath, there are a lot of efforts required to make in the establishment of an accurate numerical method for the multiple jets in the LD vessel.

This article proposes a three-dimensional mathematical model aimed to simulate the supersonic free-surrounding jets flow from a lance assumed under the converter conditions, which is verified with physical modeling data. ${ }^{12)}$ A 4De-Laval-nozzles lance with prescribed nozzle configurations was put into use with inclination angles of $10^{\circ}, 12^{\circ}$ and $14^{\circ}$. First, this computational model and its mathematical equations are described. Thereafter, the final 3D simulating results of the jet regimes as well as physical data or semi-analytical method for confirming the model are presented. Finally, the fluid flow field of characteristic parameters, the jet coalescence pattern, and the usefulness of these results on an augmented knowledge for a top-blown process are discussed.

\section{Computational Model}

\subsection{Numerical Assumptions}

In order to formulate the transport equations for this model, the following assumptions have been taken into account:
A. The gas fluid, carbon dioxide, is compressible simu- lated by the ideal gas law.
B. The gas molecular viscosity, $\mu$, is described as a function of temperature by the kinetic theory. ${ }^{13)}$
C. The flow is three-dimensional, steady-state and non- isothermal.

\subsection{Governing Equations}

In this model, the transport equations for the mass, momentums, and energy are expressed in the form of the Navier-Stokes equations according to the assumptions (A-C) given above.

$$
\begin{array}{r}
\frac{\partial\left(\rho u_{i}\right)}{\partial x_{i}}=0 \ldots \ldots \ldots \ldots \ldots \ldots \ldots \ldots \ldots \ldots \ldots \ldots \ldots \\
\frac{\partial\left(\rho u_{j} u_{i}\right)}{\partial x_{j}}=-\frac{\partial p}{\partial x_{i}}+\frac{\partial}{\partial x_{j}}\left(\sigma_{i j}\right) \ldots \ldots \ldots \\
\frac{\partial\left(\rho u_{j} c_{p} T\right)}{\partial x_{j}}=u_{j} \frac{\partial p}{\partial x_{j}}+\sigma_{i j} \frac{\partial u_{i}}{\partial x_{j}}-\frac{\partial q_{j}}{\partial x_{j}}
\end{array}
$$

Here, the necessary terms, including the viscous stress tensor $\sigma_{i j}$, the heat conduction vector $q_{j}$, the gas viscosity $\mu$, and the gas density $\rho$ used in the three expressions can be seen in the Table 1.

\subsection{Turbulence Model}

It has been proved that the calculated results are not affected by the kind of the turbulence model in the case of the

\begin{tabular}{|c|c|}
\hline Defined equations & Model parameters \\
\hline$\sigma_{i j}=\mu\left(\frac{\partial u_{i}}{\partial x_{j}}+\frac{\partial u_{j}}{\partial x_{i}}\right)-\frac{2}{3} \mu\left(\frac{\partial u_{k}}{\partial x_{k}}\right) \delta_{i j}$ & $\underline{\text { Turbulence model parameters }}$ \\
\hline$q_{j}=-k_{T} \frac{\partial T}{\partial x_{j}}$ & $\begin{array}{c}C_{l}=3.941 \text { angstrom } \\
C_{E}=195.2 \mathrm{~K}\end{array}$ \\
\hline$\mu=2.67 \times 10^{-6}\left(C_{E} / C_{l}^{2}\right) \sqrt{M / T}$ & $\sigma_{k 1}=2.0$ \\
\hline$\rho=p /(R T / M)$ & $\sigma_{\omega}=2.0$ \\
\hline$\beta_{1}=0.09\left(1+1.5 F\left(M_{t}\right)\right)$ & $\sigma_{k 2}=1.0$ \\
\hline$\beta_{2}=0.072\left(1-1.875 F\left(M_{t}\right)\right)$ & $\sigma_{\varepsilon}=1.2$ \\
\hline$F\left(M_{t}\right)=\left\{\begin{array}{lr}0 & , M_{t} \leq 0.25 \\
M_{t}^{2}-0.0625, & M_{t}>0.25\end{array}\right.$ & $C_{2}=1.9$ \\
\hline$M_{t}=\sqrt{2 k / a^{2}}, a=\sqrt{\gamma \mathrm{R} T / \mathrm{M}}$ & $\begin{array}{c}\text { Carbon dioxide } \\
c_{p}=840.37 \mathrm{~J} \mathrm{~kg}^{-1} \mathrm{~K}^{-1}\end{array}$ \\
\hline$C_{1}=\max \left[0.43, \frac{\eta}{\eta+5}\right], \eta=S \frac{k}{\varepsilon}$ & $\begin{array}{c}k_{T}=0.0145 \mathrm{~W} \mathrm{~m}^{-1} \mathrm{~K}^{-1} \\
\mathrm{M}=44 \mathrm{~kg} \mathrm{kmol}^{-1} \\
\mathrm{R}=8.314 \mathrm{~J} \mathrm{~mol}^{-1} \mathrm{~K}^{-1}\end{array}$ \\
\hline$S=\sqrt{2 S_{i j} S_{i j}}, \quad S_{i j}=\frac{1}{2}\left(\frac{\partial u_{j}}{\partial x_{i}}+\frac{\partial u_{i}}{\partial x_{j}}\right)$ & $\gamma=1.28$ \\
\hline
\end{tabular}
jet from single-nozzle lance. ${ }^{2)}$ However, it is still lack of
Table 1. Modeling terms.

conclusions to decide whether the computed results will be affected by different turbulence model or not in the case of the jets from multiple-nozzles lance. On the one hand, the standard $k-\omega$ model based on the Wilcox turbulence model ${ }^{14,17)}$ which incorporates modifications for lowReynolds-number effects, compressibility, and shear flow spreading is used. It is known that the Wilcox model predicts free shear flow spreading rates that are in close agreement with measurements for far wakes, mixing layers, plane, round, and radial jets, and is thus applicable to wallbounded flows and free shear flows. On the other hand, the theory of $k-\varepsilon$ models is widely applied in the models ${ }^{6-11)}$ so far and the realizable one ${ }^{15}$ ) among them can predict the spreading rate of the single jet accurately. Hence, it is wise to compare these two different turbulent models so that more accurate results can be shown considering the multiple-jets coalescence pattern.

In the standard $k-\omega$ model, transport equations of the turbulence kinetic energy, $k$, and the specific dissipation rate, $\omega$, are obtained by:

$$
\frac{\partial}{\partial x_{j}}\left(\rho u_{j} k\right)=\tau_{i j} \frac{\partial u_{i}}{\partial x_{j}}-\beta_{1} \rho \omega k+\frac{\partial}{\partial x_{j}}\left[\left(\mu+\frac{\mu_{T 1}}{\sigma_{k 1}}\right) \frac{\partial k}{\partial x_{j}}\right]
$$

$$
\begin{aligned}
& \frac{\partial}{\partial x_{j}}\left(\rho u_{j} \omega\right) \\
& \quad=\frac{\omega}{k} \tau_{i j} \frac{\partial u_{i}}{\partial x_{j}}-\beta_{2} \rho \omega \omega+\frac{\partial}{\partial x_{j}}\left[\left(\mu+\frac{\mu_{T 1}}{\sigma_{\omega}}\right) \frac{\partial \omega}{\partial x_{j}}\right]
\end{aligned}
$$

where the turbulent viscosity, $\mu_{T 1}$, is computed by combining $k$ and $\omega$ as $\mu_{T 1}=\rho k / \omega$. The Reynolds stress tensor, $\tau_{i j}$, is $\tau_{i j}=-\rho \overline{u_{i}^{\prime} u_{j}^{\prime}}$.

In the realizable $k-\varepsilon$ model, the transport of the turbulent kinetic energy $k$ and the dissipation rate $\varepsilon$ is given by: 


$$
\frac{\partial}{\partial x_{j}}\left(\rho u_{j} k\right)=\tau_{i j} \frac{\partial u_{i}}{\partial x_{j}}-\beta_{3} \rho \varepsilon+\frac{\partial}{\partial x_{j}}\left[\left(\mu+\frac{\mu_{T 2}}{\sigma_{k 2}}\right) \frac{\partial k}{\partial x_{j}}\right]
$$

$$
\begin{aligned}
& \frac{\partial}{\partial x_{j}}\left(\rho u_{j} \varepsilon\right) \\
& \quad=C_{1} S \rho \varepsilon-C_{2} \rho \frac{\varepsilon^{2}}{k+\sqrt{v \varepsilon}}+\frac{\partial}{\partial x_{j}}\left[\left(\mu+\frac{\mu_{T 2}}{\sigma_{\varepsilon}}\right) \frac{\partial \varepsilon}{\partial x_{j}}\right]
\end{aligned}
$$

As in the standard $k-\omega$ model, combining $k$ and $\varepsilon$, the turbulent eddy viscosity $\mu_{T 2}$ is defined by $\mu_{T 2}=C_{\mu} \rho k^{2} / \varepsilon$ in which $C_{\mu}$ is derived from the mean strain and rotation rates, the angular velocity of the system rotation, and the turbulence fields, $k$ and $\varepsilon$. All the simulating constants employed in these aforementioned transport equations are listed in Table 1.

\subsection{Computational Domain and Numerical Methodol- ogy}

To reduce the computational time of the numerical simulations, only a quarter of the total flow domain was simulated by splitting the entire domain with the two vertical midplanes of the converter. The computational domain along with the mesh used for the model of the lance which has a prescribed configuration and consists of 4 Laval nozzles is shown in Fig. 1. The geometrical data of the domain is shown in Table 2. The three-dimensional solution domain was discretized in a meshing scheme which specified that the mesh was composed primarily of hexahedral elements but included wedge elements where appropriate, i.e. the transitional area between the nozzle exit and the main cylinder domain. In this arrangement, nodes were considered at every vertex of each control volume and were the locations of scalar quantities such as pressure, density, temperature, and turbulence intensities. The computational domain for the jets was created in a cylindrical manner with a radial length of 12 nozzle exit diameters $\left(D_{o}\right)$ and an axial length of $40.2 D_{o}$, which had a total amount of about 0.79 million cells. It took about $9 \mathrm{~d}$ to reach the converged simulation results.

The jets flow from the lance reaches a supersonic velocity, which renders the influence of the variation of fluid density crucial. It is known that compressibility effects are encountered in gas flows at high velocity and in which there are large pressure variations. When the flow velocity approaches or exceeds the speed of sound of the gas or when the pressure change in the system is large, the variation of the gas density with pressure has a significant impact on the flow velocity, pressure, and temperature. Therefore, the density-based method was chosen to calculate this compressible model. In the density-based category, the continuity equation is solved for density while pressure is described via the equation state. A second-order-upwind scheme was performed as the discretization for the transport equations.

The following boundary conditions were specified, including one inlet face, two walls of the lance and its nozzles, two symmetry faces and three outlet ones. At the jets inlet boundary to the nozzle, inlet total temperature, total pressure, static pressure and turbulence parameters were specified. At the domain outlet boundary, a pressure outlet condition was applied to the three outlet faces under the
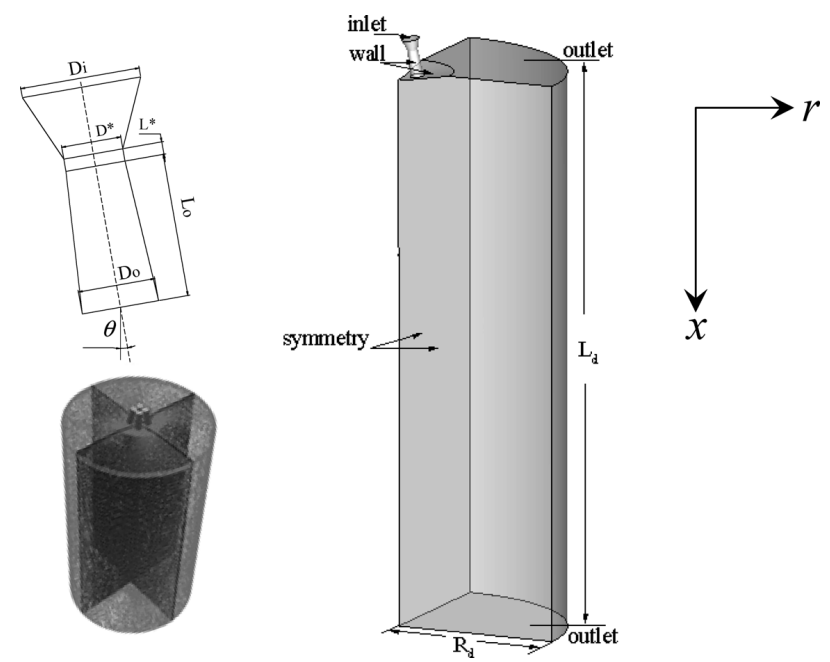

\begin{tabular}{|c|c|c|c|c|c|c|c|c|c|}
\hline \multicolumn{8}{|c|}{ Nozzle } & \multicolumn{2}{|c|}{ Domain } \\
\hline$D_{o}[\mathrm{~m}]$ & $D_{i}[\mathrm{~m}]$ & $D^{*}[\mathrm{~m}]$ & $L^{*}[\mathrm{~m}]$ & $L_{o}[\mathrm{~m}]$ & & [degree & & $D_{d}[\mathrm{~m}]$ & $L_{d}[\mathrm{~m}]$ \\
\hline 0.0496 & 0.077 & 0.0384 & 0.008 & 0.092 & $10^{\circ}$ & $12^{\circ}$ & $14^{\circ}$ & 1.2 & 2 \\
\hline
\end{tabular}

Fig. 1. Nozzle configuration and numerical domain.

Table 2. Geometrical size of model.

Table 3. Boundary conditions.

\begin{tabular}{ccccc}
\hline \multicolumn{3}{c}{ Inlet } & \multicolumn{3}{c}{ Outlet } \\
\hline$P_{i, \text { total }}[\mathrm{MPa}]$ & $P_{i, \text { static }}[\mathrm{MPa}]$ & $T_{i, \text { total }}[\mathrm{K}]$ & $P_{o}[\mathrm{MPa}]$ & $T_{o}[\mathrm{~K}]$ \\
\hline 0.784 & 0.0981 & 308 & 0.0981 & 1923 \\
\hline
\end{tabular}

commercial conditions in the vessel. A no-slip condition was applied to the walls and a standard wall function was used to model the mean velocities near the wall. All the boundary conditions are listed in Table $\mathbf{3}$.

The three dimensional solution domains were all established and discretized by the software GAMBIT ver. 2.3.16, and their calculations were performed by code of software FLUENT ver. 6.3.26.

\section{Results}

Initially, the lance with the inclination angle of $12^{\circ}$ was studied by the two turbulence models to compare the simulated results between different turbulent models. The velocity distributions along the radius in five different cross-sections by means of two modeling predictions, the standard $k-\varepsilon$ model and the realizable $k-\omega$ model, and physical modeling data in the Chen's work ${ }^{12)}$ were plotted in Figs. 2(a) and 2(b), respectively. Compared to the experimental results, the tendency of velocity variation derived by the standard $k-\omega$ model fits the physical tendency better than that by the realizable $k-\mathcal{E}$ model, though the velocity profile along the radius at each section of lance height in both turbulence models has the same variation as that in the experimental model. It is because that the interfering area within the multiple jets is in a short radial distance from the lance center concluded from the experimental data in Fig. 2(b), and the radial distance of the jets interfering area from the lance center calculated by the realizable $k-\varepsilon$ model is broader than that of the standard $k-\omega$ model. In Chen's experimental work the model was concerned with a 3-Laval- 

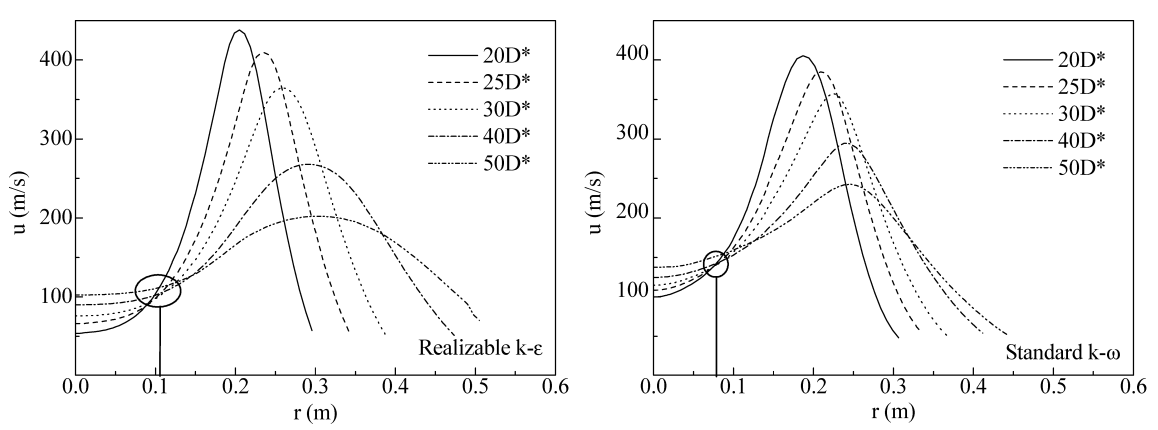

(a)

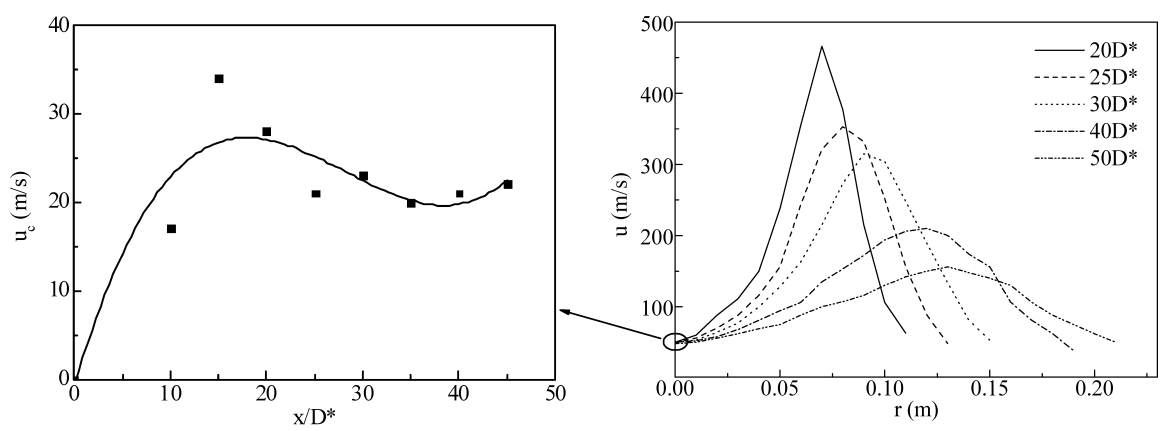

(b)

Fig. 2. Velocity profiles in the radial direction at different cross-sections. Comparison between simulated results of the $12^{\circ}$-inclination-angle lance and experimental data: (a) simulating data and (b) measured data. ${ }^{12)} D^{*}$ is the diameter of the nozzle throat and $u_{c}$ is the velocity magnitude along the axial center of the lance.

nozzle lance which is different from this three-dimensional model, but the multiple jets flow from the same gas source in the Laval nozzle lance to a free surrounding domain should conform to general variation principles according to the steelmaking experience. Therefore, this 3D model is applicable to demonstrate the behavior of multiple jets properly and in the following studies the standard $k-\omega$ model was chosen to get simulating data, considering the transitional regions from the nozzle tips: supersonic flow, transonic flow, subsonic flow and the incompressible flow. ${ }^{16)}$ The authors believe the standard $k-\omega$ model and the realizable $k-\varepsilon$ model have their own best calculation conditions such as applicable situations, domain size, calculation time step and so on. Nevertheless, the same calculation conditions have been applied for both models in the present study and it would cause the difference in the calculated results.

Typically, the hot spot (the center area of each jet within the whole jets body) geometry of the top blown jets has been assumed in an expectation of the straight procession as well as the spread with a given cone angle as a result of the difficulty of measurement for the jets behavior on a commercial basis. But each individual one of the multiple jets in the LD can digress from its straight path based on the simple geometrical calculation. In order to illustrate and analyze the inner relations of each jet and its flow field distributions, a 4-nozzle lance was simulated with inclination angles, $\theta$, of $10^{\circ}, 12^{\circ}$ and $14^{\circ}$ as an application of the present model. The final computational flow field distributions of the jets with three different inclination angles are shown in Figs. 3(a) velocity magnitude and 3(b) total temperature, respectively. Here, total temperature $T_{\text {total }}$ is the temperature at the thermodynamic state what would exist if the fluid were brought to zero velocity. On the other hand, static temperature $T_{\text {static }}$ is the temperature that is measured moving with the fluid. For compressible fluids, the total temperature

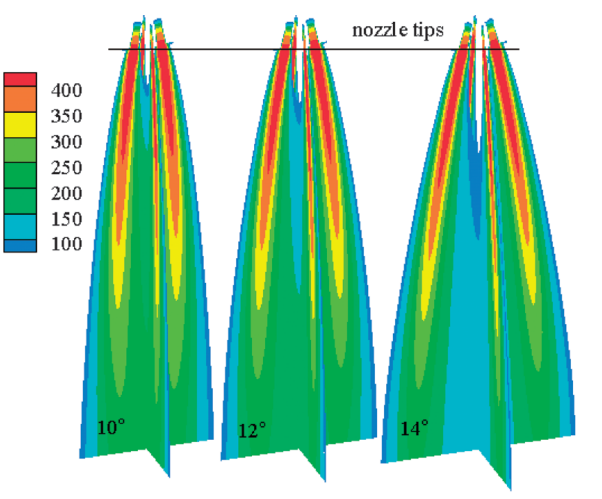

(a) Velocity magnitude $(\mathrm{m} / \mathrm{s})$

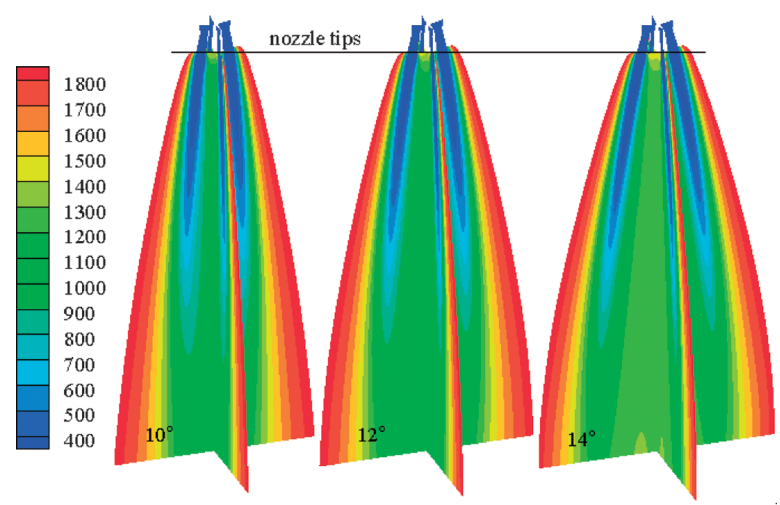

(b) Total temperature (K)

Fig. 3. Simulating results of velocity magnitude and total temperature field distributions of the three kinds of multiple jets: (a) velocity magnitude $(\mathrm{m} / \mathrm{s})$ and (b) total temperature $(\mathrm{K})$. 
is computed from the total enthalpy $H_{\text {total }}$ using the current heat capacity $C_{p}$ and the relationship between $T_{\text {total }}$ and $T_{\text {static }}$ is expressed as follows,

$$
\frac{T_{\text {total }}}{T_{\text {static }}}=1+\frac{\gamma-1}{2} M_{t}^{2}
$$

For incompressible fluids, the total temperature is equal to the static temperature.

The fluid flow distributions of characteristic parameters, velocity magnitude and total temperature indicate that the jets tend to unite by absorbing the surrounding gas. In the fourth part of this paper, these phenomena will be discussed in detail.

\section{Discussion}

\subsection{Coalescence Pattern of the Multiple Jets}

As mentioned before, the multiple jets has a distinct characteristic from the single jet, which is a tendency to converge toward the lance axis, because the multiple jets are inclined to interfere with each other and are drawn to one another. By entraining the atmospheric gas into the jets, some difference is generated among the jets and some interaction works between the jets. In this part, the multiple jets with different inclination angles were simulated to reveal the configuration of the jets coalescence behavior in the following ways. First, both the spreading laws of the jets boundary in the radial direction and the attenuation or growth laws in the axial direction of the jets were investigated, and then reasons for the multiple jets interfering behavior were analyzed, and finally a geometrical outline of the multiple jets was summarized in a comparison with the zonal structure of the single jet.

The spreading laws of the jets boundary in the radial di-

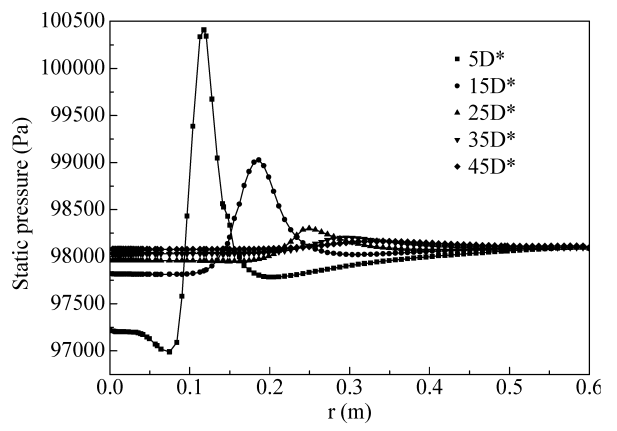

(a)

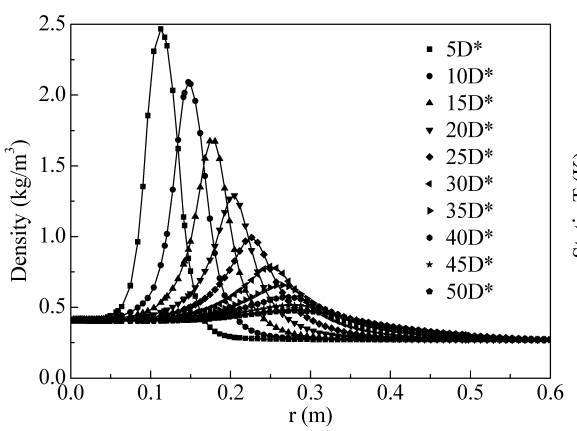

(c) rection are explained by the illustrations of jets parameters as mentioned before at different cross-sections shown in Fig. $4\left(\theta_{3}=14^{\circ}\right)$. In order to continue the clearer analysis in the subsequent parts, the jets were specified into two regions by each individual jet center: one is the inner side where the jets are near the lance center, and the other is the outer side where the jets are away from the lance axis.

In Fig. 4(a), it shows that in each cross-section the pressure of jets in the lance center is the smallest, and pressure differences occurred between the inner side and the outer side. Moreover, the pressure differences between the two sides of each jet turn into smaller and smaller with the axial procession of the jets flow, which means that the jets turn into a steady state finally. And the pressure of the jets in the inner side changes more tensely than that of the jets in the outer side. From each jet center to the two sides, Fig. 4(b) shows an inclination that the attenuation rate of the jets velocity in the inner part is slower than that in the outer part, and this inclination comes out more obviously as the axial distance from the nozzle exits increases. Further more, with the jets keeping on the procession in the axial direction, the velocity difference between the lance center and the jet center turns into smaller and smaller. In Fig. 4(c), similar to the velocity variation, the density in the inner part also has a relatively bigger value than that of the outer part; which means there are fluid mixtures in the inner part. In the consideration of the static temperature, because of the high background temperature, there is a large temperature difference happening between the jets and the ambient, and it leads temperature transmitted to the jets. That is why static temperature increases in all directions shown in Fig. 4(d), the radial one and the axial one.

Generally speaking, each jet center gradually digresses from the lance center as the flow spreading not in a straight line based on the inclination angle but in a converged way.

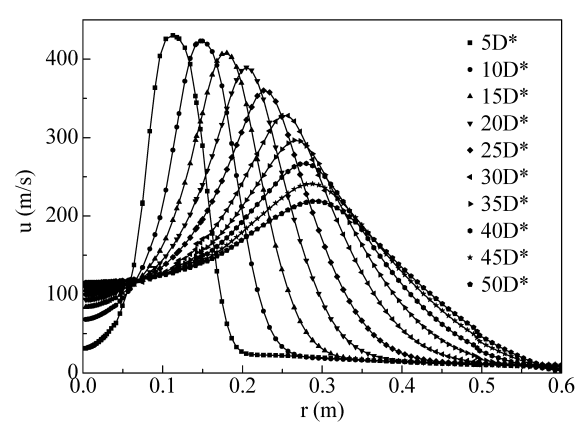

(b)

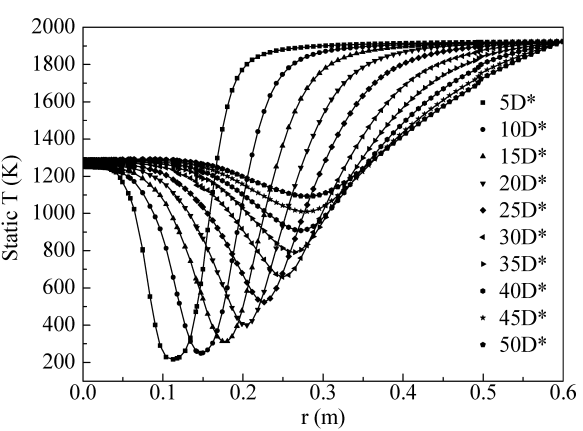

(d)

Fig. 4. Jets $\left(\theta_{3}=14^{\circ}\right)$ behavior in the radial direction on different cross-sections explained by the distributions of four parameters: (a) static pressure, (b) velocity, (c) density, and (d) static temperature. 
In each center of the individual jet, there is a reducing tendency lying in the jets parameters, static pressure, velocity, and density, while static temperature is inclined to rise in the jets axial direction of the whole jetting course. It means that the variational inclination of static pressure, static temperature, and density, in the jets center is opposite to those in the lance center.

To uncover the reasons of these phenomena is to summarize the relationship lying in these four parameters variations. According to the Coanda effect in the hydrodynamics that in free surroundings a jet of fluid entrains and mixes with its ambient gas as it flows away from a nozzle, the ambient gas was engulfed by the multiple jets quickly as soon as the jets discharged from the lance. The ambient gas in the lance center is no longer in a static state, and it means that the jets flow near the lance axis are companied by a moving fluid while the jets flow away from the lance axis still enter in a free surrounding. As flow accelerates to try balancing the momentum transfer, pressure differences across the jets are resulted, as is shown in Fig. 4(a) that the pressure in the inner part is much lower than the background pressure. Because of the absorption action, the inner part surrounded by the four fast jets becomes the area with a minus pressure. Because of the existence of these pressure differences, the jets are deflected closer to each other and eventually may unite. Finally the pressure magnitude goes up slowly and returns to the ambient pressure value. As a rule of temperature transmission, the static temperature there reduces because the surrounding gas in a high temperature mixes with the jets in a low temperature.

To summarize the coalescence patter of the multiple jets, the chart of the multiple jets turbulence structure is put forward in Fig. 5. Mach number map of the jets $\left(\theta_{2}=12^{\circ}\right)$ was plotted in Fig. 5(a) which shows that the jets tend to attract each other, and the contour of the Mach number map was charted in Fig. 5(b). With the comparison of the single jet, shown in Fig. 5(c), the regions among the multiple jets are separated into two main parts by the point where Mach number is unity in the axial direction as well. However, dissimilar to the bilateral symmetry characteristic of the single jet in the radial direction, there is an interfering area between the multiple jets called inner companied flow part, and the jets here are companied by the moving fluid. On the other side called the outer free flow part, the jets turn their direction to the lance center though they are the free surrounding parts.

\subsection{Effect of the Inclination Angles on the Multiple- jets Coalescence Pattern}

As mentioned before, the inclination angle is a main factor influencing the interfering behavior of the multiple jets. It is assumed that the jets may unite quickly in the flow domain if the extent of the nozzles inclination is small enough. In this part, the effect of the inclination angles was explained from two main aspects of the jets, which are the digression of each individual jet center and the fluid flow field distributions in the inner companied flow part.

As is well known that the Mach number is an effective dimensionless number to observe the structure of the jets flow, it is applicable to compare and analysis the Mach number contours of these jets under diverse inclination angles with the aim of illustrating their corresponding coalescence patterns. As the Mach number map of the jets with $\theta_{2}=12^{\circ}$ was shown in the former part, Fig. 5(a), Mach number maps of the other two jets were plotted in Figs. 6(a) $\theta_{1}=10^{\circ}$ and 6 (b) $\theta_{3}=14^{\circ}$, respectively. By putting the aforementioned three maps together, it is concluded that the interfering extent of the multiple jets increases with the decrease of the inclination angles. From the map in Fig. 6(a), the multiple jets exist in a distance and then disappear conforming to the assuming model for the interference behavior of multiple jets proposed by Naito et al. ${ }^{3)}$

In order to clarify the effect of the nozzle angle on the coalescence more systematically, velocity magnitude maps of the jets with $\theta_{2}=12^{\circ}$ in three planes axially distant from the nozzle tips of $0.8 \mathrm{~m}, 1.2 \mathrm{~m}$ and $1.6 \mathrm{~m}$ were plotted in Fig. 7-1 respectively. Also, velocity magnitude maps of the three kinds of jets in the plane with the distance of $1 \mathrm{~m}$ from the nozzle tips axially were charted in Fig. 7-2, respectively. In Fig. 7-1, the combination type of the jets is that each jet center decays gradually and tends to mix together. In Fig. 7-2, it reveals the aforementioned conclusion that the coalescence of the multiple jets tends to decay while the inclination angle increases.

In the inner companied flow part, the jets behaved differently in the lance center according to distinct inclination angles as shown in Figs. 8(a)-8(d). From every single plotting line, the velocity variation is that the shorter the distance from the nozzles tips is, the severer the increasing changes are, and then the velocity rises more and more slowly; similar to the velocity growth style the static pressure first rises sharply and continues a gradual growth later on. To sum up, the velocity magnitude and density increase while the static temperature decays with the decrease of the inclination angle between these three kinds of jets; and the changing style of static pressure is that its declination magnitude near the nozzle tips becomes less as the inclination angle increases.

In order to indicate the effects of the inclination angle on the jets uniting form more specifically, the velocity and static pressure profiles of the jets with $\theta_{1}=10^{\circ}$ along the radius were shown in Figs. 9(a) and 9(b), respectively. Comparing the velocity profiles in Fig. 4(b), the decline of the inclination angle can vary the jets flowing behavior to a large extent, and it shows that each individual jet is able to disappear and totally combine together to be the style of a single jet from one nozzle whose velocity profile along the radius is in the form of symmetrical Gaussian distribution as the extreme coalescence pattern.

To investigate the reasons of these phenomena is to investigate the variant property of pressure distribution in these three cases. Figure 9(b) shows that at first the static pressure in the inner part is much smaller than that in the outer part, and a smaller inclination angle can result in a relatively stronger pressure difference which leads to a tough combination between the individual jets. In summary, a smaller inclination angle produces a shorter distance between each jet at the nozzle tips, and then the jets abstracting power to each other and the engulfing power to the ambient gas become stronger, which leads to the faster companying fluid in the inner part and eventually mixing together into a whole.

\subsection{Augmenting Application in Metallurgical Process}

With this model it is possible to predict the phenomena in the cavity, though as mentioned in the introduction the focus of this paper is to develop a 3D model for the freesurrounding jets from the lance nozzles in the conditions of the converter. Since the multiple jets are the major dynamics to support the bath actions, physical and chemical, this model can be employed to foretell the potential dynamic power and the effective radius of penetration area in the 


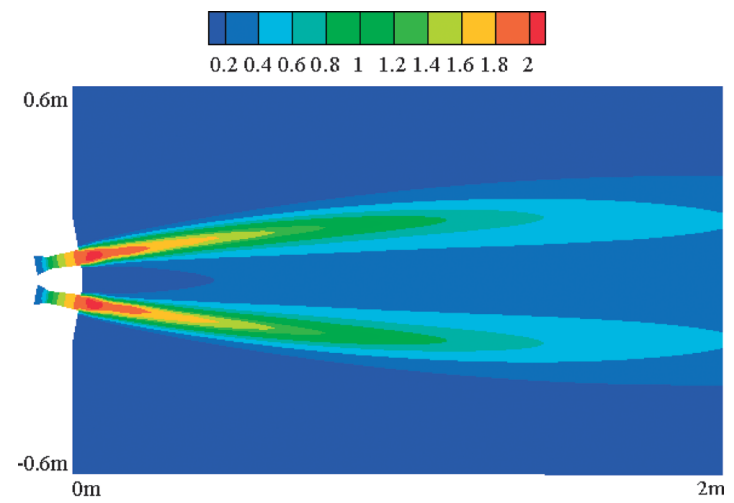

(a)

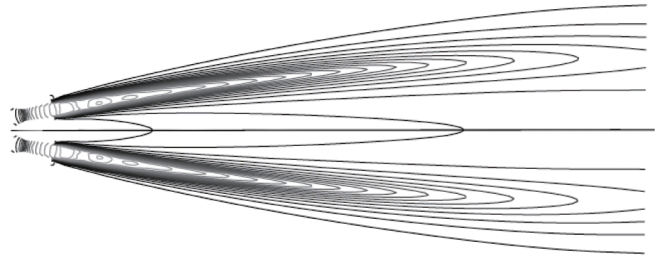

(b)

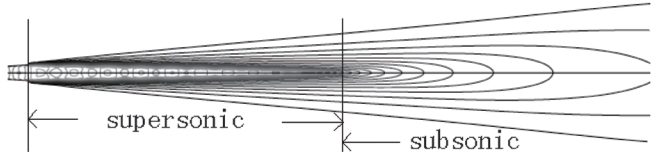

(c)

Fig. 5. Structure of the multiple jets $\left(\theta_{2}=12^{\circ}\right)$ shown by Mach number. Comparison of the configuration between the single free-surrounding jet and the multiple free jets. (a) Distribution of Mach number of the multiple jets simulated by this model, (b) diagram of the multi-jets regional structure expressed in this model, (c) diagram of the single jet regional structure designed traditionally.

vessel.

The potential dynamic power to the bath can be expressed in the form of the jet momentum, $\rho u^{2} / 2$, which is a crucial factor for the issue of studying the liquid behavior. The lance height in the LD vessel usually varies from 1 to $2 \mathrm{~m}$, and so the axial distances between $25 D^{*}$ to $50 D^{*}$ were chosen to show the jet momentum. By combining the two terms, density and velocity magnitude, the jet momentum in these 6 cross-sections were plotted in Figs. 10(a) $\theta_{1}=10^{\circ}, 10$ (b) $\theta_{2}=12^{\circ}$ and 10 (c) $\theta_{3}=14^{\circ}$, respectively. From these three graphs, the jet coalescence reduces the impact of the individual jet as the jets engulf a huge mass of ambient medium just as the imagination mentioned in the introduction. Also, there is a good correlation between the inclination angle and the interfering extent. It reveals that it is not wise to make a conclusion that the jets coalescence is detrimental without considering the axial distance of the nozzle tips to the bath surface and the radial distance to the lance axis, since the jets momentum of $\theta_{1}=10^{\circ}$ is stronger than the other two near the lance center.

In an industrial LD-process it is important to search for the information of the location and shape of the cavity since it is the place where the majority of chemical reactions and physical actions take place. With the distribution of the jet momentum, it is able to predict the location of the cavity because here it can be said that the depth of cavity, $H_{c}$, is proportional to the jet momentum, as follows:

$$
H_{c} \propto \rho u^{2} / 2
$$

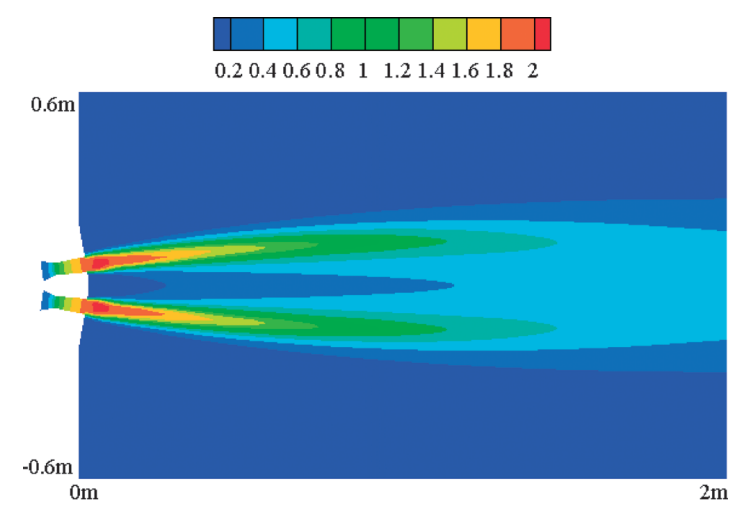

(a)

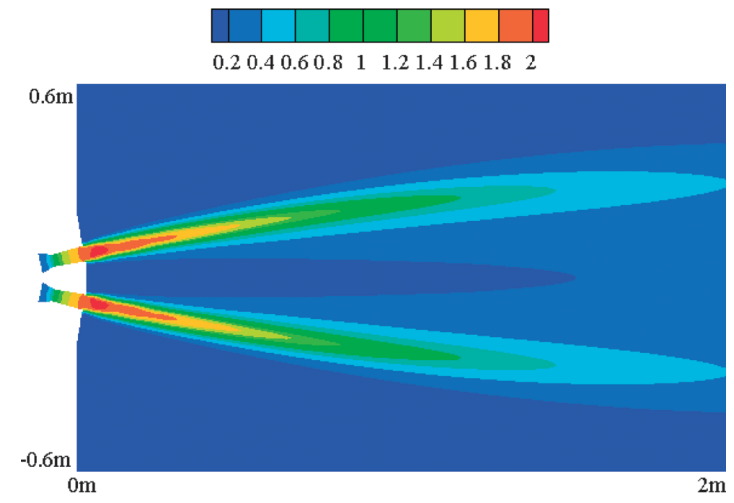

(b)

Fig. 6. Mach number maps of the two jets: (a) $\theta_{1}=10^{\circ}$, and (b) $\theta_{3}=14^{\circ}$.

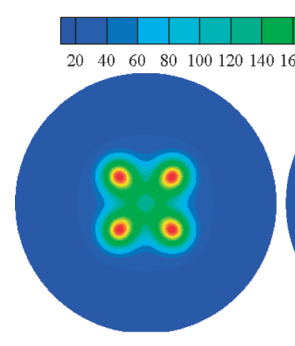

(a) $0.8 \mathrm{~m}$

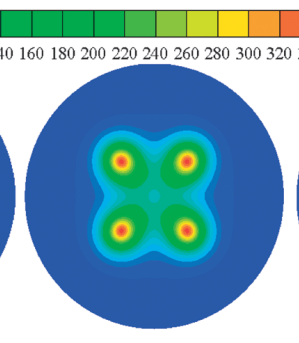

(b) $1.2 \mathrm{~m}$

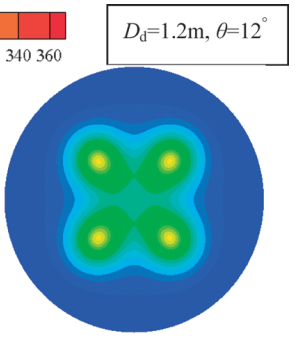

(c) $1.6 \mathrm{~m}$
(1)

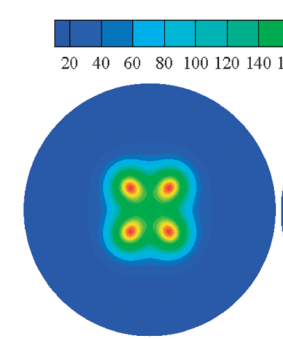

(a) $10^{\circ}$

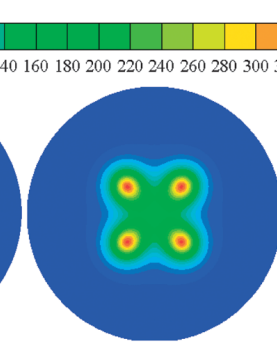

(b) $12^{\circ}$

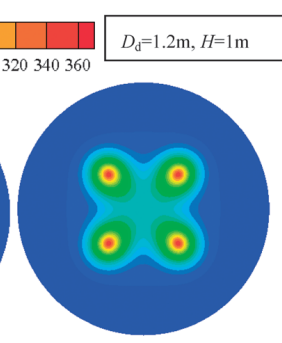

(c) $14^{\circ}$
(2)

Fig. 7. Velocity magnitude maps of the jets under diverse conditions: (1) velocity maps of the jets with $\theta_{2}=12^{\circ}$ in three planes axially distant from the nozzle tips of $0.8 \mathrm{~m}, 1.2 \mathrm{~m}$ and $1.6 \mathrm{~m}$, respectively; (2) velocity magnitude maps of three jets, $\theta_{1}=10^{\circ}, \theta_{2}=12^{\circ}$, and $\theta_{3}=14^{\circ}$, in the plane with the distance of $1 \mathrm{~m}$ from the nozzle tips, respectively. 


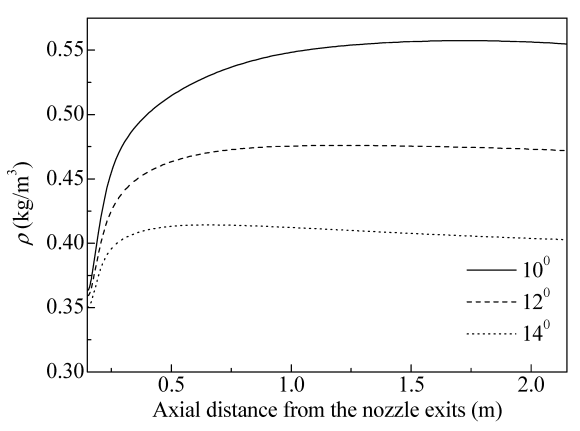

(a)

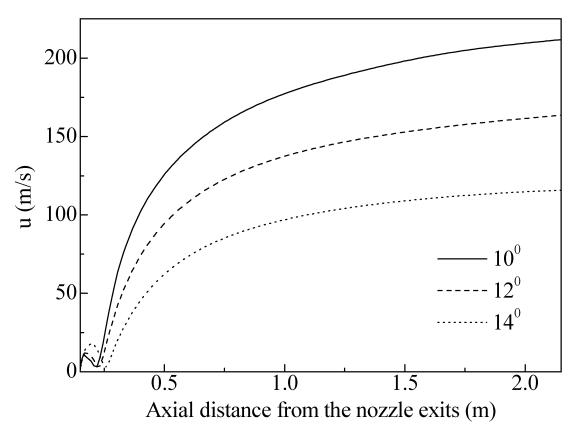

(c)

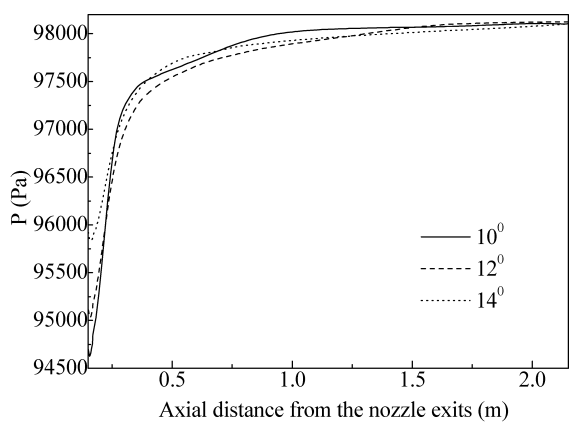

(b)

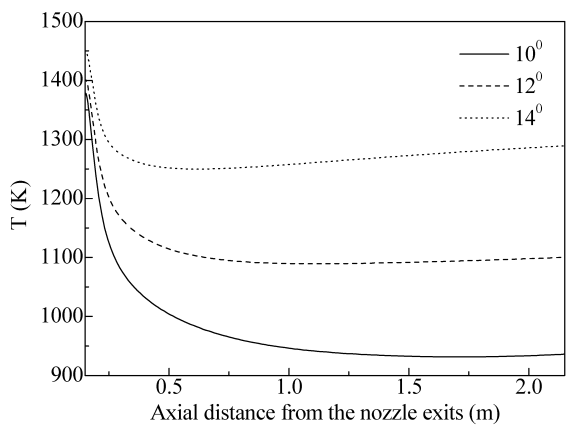

(d)

Fig. 8. Comparison of jets behaviors between three kinds of jets in the lance center. (a) density, (b) static pressure, (c) velocity magnitude, and (d) static temperature.

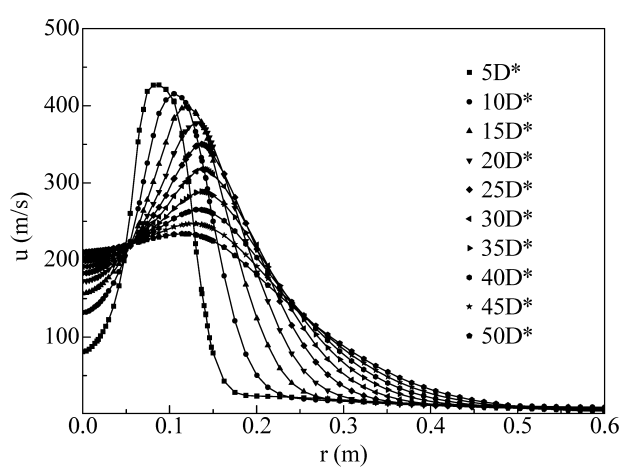

(a) velocity magnitude

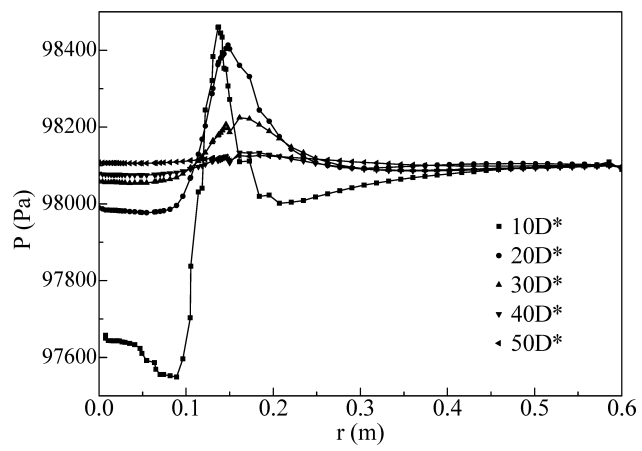

(b) static pressure

Fig. 9. Velocity and static pressure variations of the jets with $\theta_{1}=10^{\circ}$ in the radial direction of diverse cross-sections: (a) velocity magnitude and (b) static pressure.
As a rule of thumb, in the steel-making process the effective penetration from the jets to the bath is taken into account only when the velocity of the jets is $50 \mathrm{~m} / \mathrm{s}$ up, and what's more, it does harm to the converter refractory lining when the jets velocity magnitude is above 50 . Therefore, it is necessary to know the effective penetration area that is predicted in this paper with the help of the velocity magnitude 50 . The effective radius variation, $r_{e}$, of the multiple jets penetration area with three kinds of inclination angles were thus predicted in Fig. 11. It indicates that in the same lance height the effective penetration area reduces with inclination angle decreasing. The path of the center of the jets was plotted in Fig. 12 to clarify the effect of the coalescence on the jets behavior quantitatively. Though as the biggest inclination angle, the jets with $\theta_{3}=14^{\circ}$ can digress a lot from the geometrical line simple based on the calculation that the jets from the nozzle tips proceed straight without interference. Therefore, it is prudent to select the lance height from the nozzle tips to the bath surface carefully when a relatively big inclination angle is considered to be used.

In conclusion, as the lance height changing longer and longer, it could be a sign of the cavity variation that the penetration area increases while the penetration depth decreases, combining Figs. 10 and 11 together. Each effective penetration radius, $r_{e}$, displays a regular variation, shown in Fig. 11, when the inclination angle, $\theta$, and the axial distance from the nozzle tips, $H_{l}$, are only taken as arguments. With the help of this simulation model, a mathematical model was proposed shown in function (9) to depict the effects of the multiple jets coalescence on the effective penetration radius and area as well. The radial distance of each jet center digressing from its respective center path based on simple geometrical calculation, $\Delta r$, is also in a regular variation with $\theta$ and $H_{l}$ by analyzing Fig. 12. As a result, 


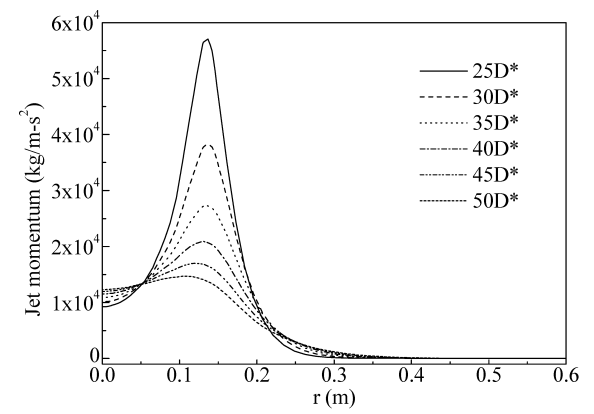

(a) $\theta_{1}=10^{\circ}$

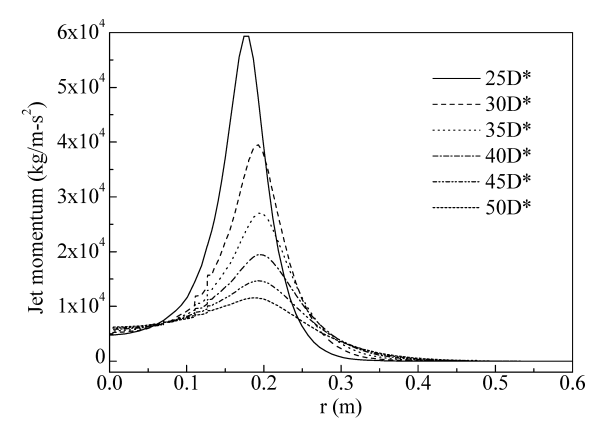

(b) $\theta_{2}=12^{\circ}$

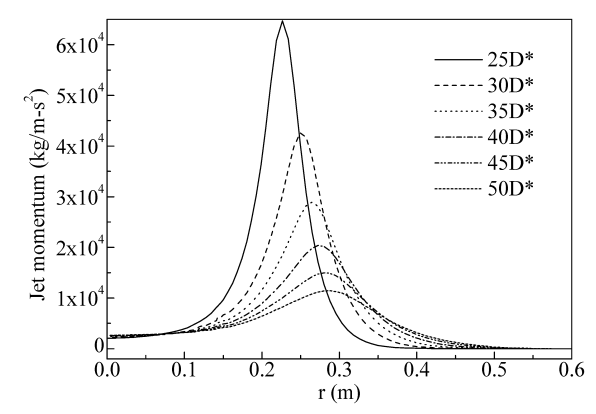

(c) $\theta_{3}=14^{\circ}$

Fig. 10. Variation of the jets momentum along the radius in 6 different cross-sections. Comparison between the jets with three kinds of inclination angles: (a) $\theta_{1}=10^{\circ}$, (b) $\theta_{2}=12^{\circ}$, and (c) $\theta_{3}=14^{\circ}$.

the variation of $\Delta r\left(H_{l}, \theta\right)$ can be also fitted into function (9) with the help of the data fitting of Fig. 12.

$$
Y=f_{1} \exp \left(H_{l} / f_{2}\right)+f_{3} \ldots
$$

where $Y$ means the variables of $r_{e}$ and $\Delta r$, and $f_{i}(\theta)$ $(i=1,2,3)$, the effects of $\theta$ on $Y$, has a formulation as $f_{i}(\theta)=c_{1}\left(\theta-c_{2}\right)^{2}+c_{3}$ by the data fitting. Here, the parameters of $c_{i}(i=1,2,3)$ that may imply the other influencing factors, such as the nozzle throat diameters, Mach number, density, operation pressure of the nozzle inlet, and so on, were considered to be constant derived from the data fitting as shown in Table 4. Therefore, based on the mathematical model in function (9), the more accurate depictions of $\Delta r$ and $r_{e}$ are applicable to develop into establishment in the future.

From an applicable point of view, it would be of interest to handle the dynamic power and the jet momentum in order to control and optimize the fluid flow behavior in the BOF. It is the author's belief that if the current simulating model is extended to also include treatments of the sur-

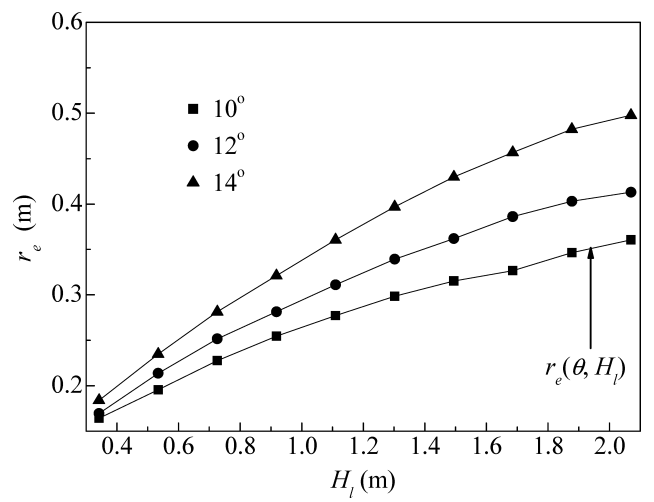

Fig. 11. Prediction of the effective radius variation of the jets penetration radius in the axial direction.

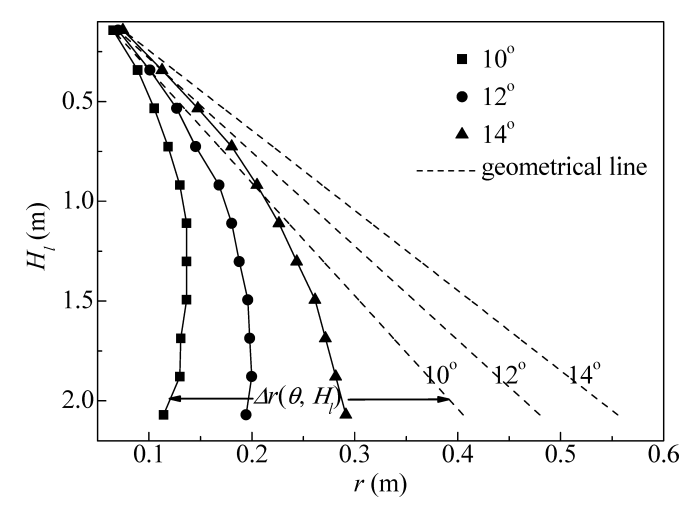

Fig. 12. Effect of the coalescence on the jets behavior.

Table 4. Constants of the mathematical model.

\begin{tabular}{ccccccc}
\hline & \multicolumn{3}{c}{$r_{e}\left(H_{l}, \theta\right)$} & \multicolumn{3}{c}{$\Delta r\left(H_{l}, \theta\right)$} \\
\hline & $c_{1}$ & $c_{2}$ & $c_{3}$ & $c_{1}$ & $c_{2}$ & $c_{3}$ \\
\cline { 2 - 7 } $10^{\circ}$ & 0.05441 & 11.783312 & -0.630965 & 0.00145 & 13.69655 & 0.097636 \\
$12^{\circ}$ & 0.06186 & 12.05779 & -1.81536 & 0.01625 & 12.64985 & 1.49764 \\
$14^{\circ}$ & -0.0515 & 11.80379 & 0.70449 & -0.00155 & 13.65806 & -0.11236 \\
\hline
\end{tabular}

roundings of the converter wall and reactions happening in the converter just as proposed in the literatures ${ }^{8-10,17)}$ it would be possible to establish a complete simulating model of a top-blown process. In this way, a final and whole 3D model of a top-blown bath would be applicable to combine with experimental data from laboratory experiments and plant experiments to augment the information of the mechanisms of fluid flow style and reactions in the vessel, such as the penetration, splashing, and decarburization.

\section{Conclusion}

A three dimensional model of the top-blown multiple jets has been presented to investigate the coalescence pattern of the free surrounding jets and the effects of inclination angles on this coalescence pattern. With the multiple jets assuming under a commercial conditions of high temperature, different Mach numbers, and high inlet pressure, the overall conclusion is that it is promising to understand the multiple jets behavior in a top-blown converter and extrapolate the cavity formation in the LD bath.

Specific conclusions from the top-blown compressible 
jets simulation include:

(1) Turbulent anisotropic spreading of the multiple jets can not be neglected. There is a reasonably good match between the velocity radial variation laws gained by the experiments in the literature ${ }^{12)}$ and the numerical results obtained with the standard $k-\omega$ turbulent model, while the realizable $k-\varepsilon$ turbulence model resulted a relatively larger digression from the physical law.

(2) In general, each jet center gradually digresses from the lance center as the flow spreading not in a straight line based on the inclination angle but in a converged way. In each center of the jets, there is a reducing tendency lying in the jets parameters, static pressure, velocity, and density, while static temperature is inclined to rise in the jets axial direction of the whole jetting course, and it is opposite to those in the lance center.

(3) With the jets tending to attract each other and involving the ambient gas, dissimilar to the bilateral symmetry characteristic of the single jet, there is an interfering area between the multiple jets called inner companied flow part, while the other side is named the outer free flow part.

(4) The lower inclination angle leads to the jets coalescence more likely. An extreme and complete interfering pattern of the multiple jets were illustrated by the simulation of the multiple jets.

(5) A mathematical model was proposed, depicting how the variables of the effective penetration radius and the radial distance of each jet center digressing from its respective center path based on simple geometrical calculation change by taking the inclination angles and the axial distance from the nozzle tips as arguments.

(6) It is possible to use extrapolation of the jet momentum and the effective penetrating area variational laws to estimate the formation of the cavity in the BOF bath and to protect the lining of the vessel.

\section{Nomenclature}

$\mu:$ Molecular viscosity $\left[\mathrm{kg} \mathrm{m}^{-1} \mathrm{~s}^{-1}\right]$

$v$ : Kinematic viscosity $\left[\mathrm{m}^{2} \mathrm{~s}^{-1}\right]$

$\rho:$ Gas density $\left[\mathrm{kg} \mathrm{m}^{-3}\right]$

$k$ : Turbulence kinetic energy $\left[\mathrm{m}^{2} \mathrm{~s}^{-2}\right]$

$\omega:$ Specific dissipation rate $\left[\mathrm{s}^{-1}\right]$

$\varepsilon$ : Turbulence dissipation rate $\left[\mathrm{m}^{2} \mathrm{~s}^{-3}\right]$

$\mu_{T 1}$ : Turbulent eddy viscosity, also $\mu_{T 2}\left[\mathrm{~kg} \mathrm{~m}^{-1} \mathrm{~s}^{-1}\right]$

$u_{i}$ : Velocity component $\left[\mathrm{m} \mathrm{s}^{-1}\right]$

$c_{p}$ : Specific heat at constant pressure $\left[\mathrm{J} \mathrm{kg}^{-1} \mathrm{~K}^{-1}\right]$

$c_{v}$ : Specific heat at constant volume $\left[\mathrm{J} \mathrm{kg}^{-1} \mathrm{~K}^{-1}\right]$

$k_{T}$ : Thermal conductivity coefficient $\left[\mathrm{W} \mathrm{m}^{-1} \mathrm{~K}^{-1}\right]$

$\delta_{i j}$ : The Kronecker delta

$\sigma_{k 1}$ : Turbulent Prandtl numbers for $k$, also $\sigma_{k 2}$

$\sigma_{\varepsilon}$ : Turbulent Prandtl numbers for $\varepsilon$, also $\sigma_{\omega}$ for $\omega$

$C_{l}$ : Lennard-Jones characteristic length $[\AA]$
$\mathrm{M}: \quad$ Molecular weight $\left[\mathrm{kg} \mathrm{kmol}^{-1}\right]$

$C_{E}$ : Lennard-Jones energy parameter [K]

$\mathrm{R}$ : Gas constant $\left[\mathrm{J} \mathrm{mol}^{-1} \mathrm{~K}^{-1}\right]$

$\gamma:=c_{p} / c_{v}$

$M_{t}$ : Turbulent Mach number

$a$ : Speed of sound $\left[\mathrm{m} \mathrm{s}^{-1}\right]$

$D_{o}$ : Nozzle exit diameter $[\mathrm{m}]$

$D_{i}$ : Nozzle inlet diameter $[\mathrm{m}]$

$D^{*}$ : Nozzle throat diameter $[\mathrm{m}]$

$D_{d}$ : Computational domain diameter [m]

$L^{*}$ : Nozzle throat length [m]

$L_{o}: \quad$ Nozzle exit length [m]

$L_{d}$ : Computational domain length [m]

$P_{i, \text { total }}:$ Gas inlet total pressure [MPa]

$P_{i, \text { static }}:$ Gas inlet static pressure [MPa]

$T_{i, \text { total }}: \quad$ Gas inlet total temperature $[\mathrm{K}]$

$P_{o}:$ Outlet boundary pressure $[\mathrm{MPa}]$

$T_{o}$ : Outlet boundary temperature $[\mathrm{K}]$

$\theta:$ The inclination angle [degree]

$H_{c}$ : Depth of cavity [m]

$H_{l}$ : axial distance from the nozzle tips [m]

$r_{e}:$ effective penetration radius [m]

$r$ : radial distance from the nozzle center [m]

$\Delta r:$ radial distance of each jet center digressing from its respective center path based on simple geometrical calculation $[\mathrm{m}]$

\section{REFERENCES}

1) Y. Tago and Y. Higuchi: ISIJ Int., 43 (2003), 209.

2) Z. Yuan, X. Yang, Z. Lu, J. Huang, Y. Pan and E. Ma: J. Iron Steel Res. Int., 14 (2007), 01.

3) K. Naito, Y. Ogawa, T. Inomoto, S. Kitamura and M. Yano: ISIJ Int., 40 (2000), 23.

4) Y. Higuchi and Y. Tago: ISIJ Int., 41 (2001), 1454.

5) Y. Higuchi and Y. Tago: ISIJ Int., 43 (2003), 1410.

6) N. Hatta, H. Fujimoto, R. Ishii, Y. Umeda and J. Kokado: ISIJ Int., 29 (1989), 911

7) Y. Peng and T. Han: ISIJ Int., 36 (1996), 263.

8) M. Ersson, A. Tilliander, L. Jonsson and Pär Jönsson: ISIJ Int., 48 (2008), 377.

9) M. Ersson, L. Höglund, A. Tilliander, L. Jonsson and Pär Jönsson: ISIJ Int., 48 (2008), 147.

10) H. J. Odenthal, J. Kempken, J. Schlüter and W. H. Emling: Iron Steel Technol., 4 (2007), 71.

11) R. Sambasivam, S. N. Lenka, F. Durst, M. Bock and S. Chandra: Metall. Mater. Trans. B, 38B (2007), 45.

12) Y. Chen, T. Wang, L. Liu and Y. Sheng: Journal of Beijing University of Iron and Steel, 3 (1982), 12.

13) J. O. Hirschfelder, C. F. Curtiss and R. B. Bird: Molecular Theory of Gases and Liquids, John Wiley \& Sons, New York, (1954), 478.

14) D. C. Wilcox: AIAA J., 30 (1992), 2639.

15) T. H. Shih, W. W. Liou, A. Shabbir, Z. Yang and J. Zhu: Comput. Fluids, 24 (1995), 227.

16) K. Javadi, M. Darbandi and M. Taeibi-Rahni: Comput. Fluids, 37 (2008), 747.

17) X. Yang, Z. Yuan, Z. Lu and Y. Pan: CAMP-ISIJ, 20 (2007), 826. 